\title{
Estrategias de la industria cultural mexicana para el desarrollo regional del norte de Jalisco. El caso de la artesanía huichol
}

José G. Vargas-Hernández *

\begin{abstract}
Resumen: Las industrias culturales han tomado un papel muy importante en la preservación de la cultura e identidad nacional, por lo que el impulso de las mismas es un factor a considerar como parte del crecimiento económico de los países. Además de que la actividad artesanal como parte de la industria cultural juega un papel clave dentro las economías modernas. El presente estudio tiene como objetivo analizar la importancia de la producción artesanal en la zona norte del estado de Jalisco al ser promovida con fines de atractivo turístico regional, con fines culturales, económicos y sociales. En este trabajo se darán a conocer alternativas estratégicas que tanto el Estado como otras instituciones pueden implementar para impulsar el desarrollo regional, mediante la implementación de proyectos que involucren la promoción cultural y artesana de la región norte de Jalisco.
\end{abstract}

Palabras clave: Artesanías. Desarrollo regional. Industrias culturales.

\section{Estratégias culturais mexicanos para regional desenvolvimento industrial do norte de Jalisco.} Caso de ofícios huichol

Resumo: As indústrias culturais têm tido um papel muito importante na preservação da cultura e da identidade nacional, de modo que o seu estímulo é um fator a ser considerado como parte do crescimento econômico dos países. Além do ofício em si, como parte da indústria cultural desempenha um papel fundamental nas economias modernas. O presente estudo tem como objetivo analisar a importância da produção artesanal no norte do estado de Jalisco para ser promovida com a finalidade de atração turística regional, cultural, econômica e social. Neste artigo busca-se apresentar alternativas estratégicas que o Estado e outras instituições podem implementar para promover o desenvolvimento regional, através da implementação de projetos que envolvam a promoção da cultura e do artesanato da região norte de Jalisco.

Palavras-chave: Artesanato. Desenvolvimento regional. Indústrias culturais.

\section{Mexican cultural industry strategies for regional development in the northern Jalisco. The case of crafts huichol}

\begin{abstract}
Cultural industries have taken a very important role in the preservation of culture and national identity, so that the momentum of these is a factor to consider as part of the country's economic growth. In addition to the craft as part of the culture industry plays a key role in modern economies. The present study aims to analyze the importance of craft production in the northern state of Jalisco to be promoted for the purpose of regional attraction, for cultural, economic and social. This work will be announced strategic alternatives that both the State and other institutions can implement to promote regional development by implementing projects involving the promotion of culture and crafts of the northern region of Jalisco.
\end{abstract}

Keywords: Handicrafts. Regional development. cultural industries.

Classificação JEL: D20, L30, Q10.

\footnotetext{
* Professor do "Centro Universitario de Ciencias Económico Administrativas. Universidad de Guadalajara”. E-mail: jgvh0811@yahoo.com; jvargas2006@gmail.com; jgvh0811@yahoo.com, josevargas@cucea.udg.mx.
} 


\section{Introducción}

México, es un país multicultural con una gran y vasta riqueza en cuanto al patrimonio arqueológico, histórico y artístico se refiere, posee un significante número de culturas en su sociedad, que si bien no conviven entre ellas, tratan de coexistir manteniendo sus diferencias. Además de que cada una de estas distintas culturas, tienen sus propias costumbres, tradiciones e ideas; y poseen una forma distinta y característica para expresarlas.

Una de las características fundamentales que diferencia una cultura de otra en México, se puede observar en la producción artesanal, la cual varía desde los alebrijes y el trabajo del barro negro en Oaxaca, la talavera poblana, los bellos y tradicionales rebozos de San Luis Potosí, y el arte huichol de los estados de Nayarit, el norte de Jalisco y algunas zonas de Zacatecas; entre otras artesanías.

La artesanía, como parte de las industrias culturales, se ha convertido en un factor importante a considerar en las economías modernas; ya que no solo contribuyen con el crecimiento económico de los países, generando empleo e ingresos para miles de familias, si no que también ayudan a transmitir y mantener las raíces culturales e identidad de las naciones; y es un factor a considerar para un turismo que gusta de apreciar las expresiones populares del arte en diversos materiales, (Rivera Cruz, Alberti Manzanares, Vázquez García y Mendoza Ontiveros, 2008). Por ello, la artesanía huichol hecha en el norte del estado de Jalisco que comprende tablas de estambre (nierikas), figuras cubiertas de chaquira y cruces tejidas de estambre, entre otros objetos, es reconocida por su belleza y simbología contenida; y es una forma de expresión artística que refleja los sentimientos y creencias del pueblo que se llama a si mismo wixárika. En este orden de ideas podemos formular el siguiente cuestionamiento, ¿el impulso y comercialización de la artesanía huichol en la zona norte del estado de Jalisco puede contribuir al desarrollo económico regional?

Los objetivos de este artículo analítico exploratorio es hacer una revisión conceptual referente a las industrias culturales y las artesanías, estudiar el impacto económico de las industrias culturales y la artesanía, y analizar la importancia de la producción artesanal en la zona norte del estado de Jalisco al ser promovida con fines de atractivo turístico regional, con fines culturales, económicos y sociales.

\section{Antecedentes teóricos y revisión de literatura}

Hoy en día la globalización está presente en la gran mayoría de los ámbitos de la vida cotidiana. Este creciente proceso de globalización ha tendido a erosionar la soberanía de los Estados nacionales, sobre todo en el ámbito económico, pero también en el cultural (Pescador, 2008). El incesante crecimiento y avance de las tecnologías y de los medios de comunicación han 
hecho parecer al mundo cada vez más pequeño, además pareciera que se está uniformando en un solo modelo. En este orden de ideas, muchos países comenzaron a hacer una reflexión relacionada a formar parte del mundo globalizado para alcanzar mayor crecimiento, pero al mismo tiempo rescatar la importancia de mantener su identidad nacional, es decir, conservar lo que los diferencia unos de otros, lo que los hace únicos, rescatando su cultura, sus tradiciones, sus raíces.

A consecuencia de esta concientización hecha por los países se comienza a rescatar la cultura nacional, pero a la vez se le comienza a dar un carácter industrial a la producción de la misma, por lo que a mediados de los años cuarenta se utilizó el término de industria cultural, para explicar un cambio en el proceso de transmisión de la cultura, donde se hace referencia de la potencia de la radio, el cine y la naciente televisión (Theodor Adorno y Max Horkehimer, 1949).

Por otra parte las Industrias Culturales se definen como una combinación de creación, producción y comercialización de contenidos que son intangibles y que al mismo tiempo son culturales en su naturaleza, considerando que están protegidos por el derecho de autor y pueden tomar forma de bienes o servicios (Piedras, 1994). La industria cultural comprende, por lo general, los sectores editorial, multimedia, audiovisual, fotográfico, producciones cinematográficas, artesanía y diseño.

La UNESCO $(1995,2011)$ sostiene que existe una industria cultural cuando los bienes y servicios culturales se producen, reproducen, conservan y difunden según criterios industriales y comerciales, es decir en serie y aplicando estrategias de tipo económico. La acción de la UNESCO consiste esencialmente en fortalecer las capacidades locales y el acceso a mercados mundiales de países emergentes gracias a nuevas asociaciones, la ayuda de expertos, la formación, la lucha contra la piratería y el esfuerzo de la solidaridad internacional en todas sus formas.

Por su parte, Zallo (1988) se refiere a las industrias culturales como el conjunto de ramas industriales productoras y distribuidoras de mercancías con contenido simbólico, concebidas gracias a un trabajo donde interviene la creatividad e imaginación, organizadas por un capital que se valoriza y destinadas a un mercado de consumo, con fines de producción ideológica y social.

La clasificación de las Industrias Culturales según el Observatorio de Cultura y Economía (2011) está dada en nueve grupos, los cuales comprenden: 1) Artes escénicas: música, danza, marionetas, circo y ópera; 2) Audiovisuales: cine, TV y radio; 3) Servicios creativos: Arquitectura, publicidad, servicios y cultura; 4) Media interactiva: contenidos digitales, software, video juegos, animaciones; 5) Diseño: interiores, gráficos, modas y joyería; 6) Literatura y publicaciones: libros, prensa y otras publicaciones; 7) Artes visuales: pintura, escultura, fotografía y antigüedades; 8) Patrimonio cultural: museos, bibliotecas, sitios arqueológicos; 9) Conocimiento tradicional: festivales, artesanías. 
Las industrias culturales han tomado un gran auge, tanto a nivel internacional, como a nivel nacional; según estudios internacionales desarrollados por la ONU en 1998 acerca de las aportaciones económicas derivadas de la industria cultural, en México 1.5 millones de personas vivían de la promoción cultural, lo cual representó una contribución al producto interno bruto del $6.7 \%$ en ese momento (Alba, 2008).

En Europa por ejemplo las industrias culturales generan el 4.5\% del PIB europeo, además de que se valora en 1800 millones de euros los que la Unión Europea destinará a la financiación de proyectos culturales en el periodo 2014-2020 (Santander, 2012).

De acuerdo con la UNESCO (2004) en cuanto al ámbito de la producción artesanal como parte de las industrias culturales, algunos países industrializados otorgan mucha importancia a las artesanías, un ejemplo de ello se encuentra en Italia, que en el año 2001 se estima que el $24 \%$ de sus empresas eran artesanales, además de que la exportación de las artesanías representaba el 17\% del PIB. En Colombia la producción de objetos artesanales produjo unos 400 millones de dólares, además de que las exportaciones de esos objetos ascendieron a 40 millones de dólares. México, por su parte, de acuerdo con el FONART, contaba con 2.8 millones de artesanos de tiempo completo, además de 4 millones más de tiempo parcial, lo que hace un total de 6.8 millones de artesanos que operaban en México con ingresos promedio de 320 pesos por mes.

En México, por ejemplo, el fideicomiso para fomentar la producción artesanal, se da a partir de que el Estado reconoce la gran potencialidad que tenía la artesanía como salvación económica, además de que se extendió su labor a todos los lugares donde se producían artesanías, donde interviniera la producción, el financiamiento, y en una forma considerable, la comercialización de las mismas (Novelo, 1976).

El artesano, tal como se conoce actualmente, tiene su origen en las sociedades europeas, donde se desarrollaron como una parte de la sociedad que se especializaba en diferentes oficios con el fin de producir tanto objetos cotidianos como de lujo, para el resto de la sociedad.

Por otra parte en México existieron gremios de artesanos en la Nueva España, quienes fueron ordenados de manera rígida, además de que los talleres de artesanos eran explotados por los españoles, obteniendo mano de obra indígena calificada de manera gratuita.

Novelo (1993) hace referencia que fue en siglo XVII cuando ya había comerciantes dedicados a financiar la producción artesanal en las ciudades, mientras que los artesanos indígenas solo se limitaban al comercio local o regional.

Con el paso del tiempo el interés por los objetos producidos por los indígenas fue en aumento, por lo que paso a ser objeto de trabajo del Departamento Autónomo de Asuntos Indígenas, y posteriormente del Instituto Nacional Indigenista en 1948. 
Acerca de la definición de artesanía se encuentra lo que propone la $\operatorname{UNESCO}(1995,2001)$ "Los productos artesanales son los producidos por artesanos, ya sea totalmente a mano, o con la ayuda de herramientas manuales o incluso de medios mecánicos, siempre que la contribución manual directa del artesano siga siendo el componente más importante del producto acabado” (...).

Cabe señalar que es la UNESCO la única organización internacional con una visión global del papel social, económico y cultural de la artesanía en la sociedad. Por lo que se ha dedicado a desarrollar proyectos relacionados al impulso artesanal, con el objetivo de demostrar a las autoridades de diferentes naciones, estados y localidades la prioridad que merece la artesanía en los programas de desarrollo.

Novelo (1993) hace referencia al término artesanal como una peculiar manera en la que el hombre ha usado los instrumentos de trabajo para producir algo, generalmente un bien, un objeto de consumo, y que se ha relacionado con otros hombres, también de formas peculiares tanto en la producción como en la distribución y cambios de esos bienes.

Por su parte Pasteur (2004) menciona que las artesanías tradicionales son aquellos bienes realizados para el consumo doméstico y/o ritual, siendo objetos portadores de un valor histórico, cultural, utilitario o estético y que cumplen con una función socialmente reconocida, realizados por el artesano, individual o colectivamente, mediante técnicas simples.

La gran variedad de criterios que se pueden utilizar para definir a las artesanías se relaciona a que normalmente se habla de ellas como resultado de un proceso, y no como el proceso en sí (Novelo, 1976). Se considera importante explicar el proceso de producción de las artesanías, ya que uno de los criterios para definir el concepto tiene que ver con el trabajo manual en la elaboración del producto.

En este orden de ideas, la artesanía hecha por los huicholes, uno de los cuatro grupos indígenas que habitan en la región conocida como el Gran Nayar, en la Sierra Madre Occidental, es una de las más representativas de México, por su belleza y colorido, además de su simbología y diseños que datan de hace siglos.

Las principales artesanías hechas por los huicholes, son tablas de estambre, las cuales se caracterizan por sus diseños psicodélicos, que surgen a raíz de alucinaciones de los artesanos al consumir peyote, las mascaras y figuras cubiertas con cuentas de vidrio, chaquira, que representan deidades y animales sagrados, también están las cruces tejidas de estambre, las cuales representan al cosmos con cinco rumbos, cuatro puntos cardinales y el centro. Además se pueden encontrar morrales bordados a mano, aretes, collares, anillos, pulseras, etc. hechos de chaquira, que aunque no constituyen objetos rituales, forman parte de la artesanía huichol. 
Dicha artesanía puede utilizarse como atractivo y dar pie al turismo cultural en la zona norte de Jalisco, además de que puede llevarse a cabo su comercialización, para contribuir a la economía regional y principalmente a la economía familiar.

\section{Comercialización de la artesanía, visión basada en la industria y en recursos y capacidades}

Hablar de competitividad es hablar de un enfoque desde el punto de vista de ventaja competitiva. De acuerdo con Porter, para crear ventaja es necesario percibir las nuevas formas de competencia, además de asumir riesgos y hacer inversiones para ponerlas en práctica. De esta manera, la ventaja competitiva se origina de la forma en que las empresas organizan y llevan a cabo actividades discretas. Por lo que las empresas crean valor para sus compradores por medio de la realización de estas actividades (Porter, 1985).

La ventaja competitiva a la que Porter hace referencia, puede verse reflejada en tres aspectos fundamentales, el liderazgo en costos, la diferenciación y el enfoque. El liderazgo en costos se centra en los bajos costos y bajos precios para atraer más clientes, por lo que con respecto a este factor es importante considerar que los artesanos huicholes tienen el poder de fijar precios a sus artesanías, lo cual, los puede establecer como productores de bajo costo y convertirlos en líderes en este ramo. La diferenciación, se basa en la entrega de productos de tal forma que los consumidores los encuentren valiosos y diferentes. Las formas de expresión artística huichol reflejan sus sentimientos religiosos, que plasman en variedad de objetos, diseños de ropa, en la construcción de sus templos e instrumentos musicales, etc.. Lo cual hace a la artesanía huichol totalmente diferenciable y única de otras artesanías, ya sea hechas en la misma zona norte del estado de Jalisco o en el resto del país. Por último, el enfoque, que cubre las necesidades de un segmento particular o nicho del mercado, aspecto importante a considerar ya que dependiendo a quien se quiera dirigir el producto artesanal se tienen que tomar en cuenta elementos de clase, estética y tradicionalidad (Novelo, 1993).

Según Peng (2006), las estrategias de una empresa deben fundamentarse en acciones previamente planeadas y en actividades emergentes que no lo están. Sin embargo el diseño de dichas estrategias debe realizarse mediante una valoración de fuerzas (F) y debilidades (D), además de las oportunidades (O) y amenazas (A) del entorno en que se desenvuelven. En este sentido instituciones como el Fondo Nacional para el Fomento de las Artesanías (FONART), la Comisión Nacional para el Desarrollo de los Pueblos Indígenas (CDI), el Consejo Nacional para la Cultura y las Artes (CONACULTA), que son instituciones dedicadas al fomento artesanal y cultural en México, con objetivos de impulsar la actividad artesanal, para de esta forma contribuir al ingreso 
familiar de los artesanos, mediante su desarrollo humano, social y económico; son quienes deben hacer este tipo de análisis estratégico como apoyo al artesano huichol.

Las estrategias de comercialización de la artesanía huichol deben llevarse a cabo por parte de las instituciones ya mencionadas, además de que las autoridades municipales y estatales también tienen que participar activamente en esta tarea; ya que son estas instituciones y el gobierno quienes tienen los conocimientos y medios necesarios para ayudar a los huicholes a posicionar sus productos en el mercado. Es muy importante tomar en cuenta esta consideración ya que el pueblo indígena huichol no tiene un conocimiento en áreas como la organización, gestión, promoción, mercadeo, ventas, etc. Y debido a esto les es difícil desenvolverse en el mundo de los negocios.

Al utilizar herramientas básicas de estrategia, es fácil notar que la artesanía huichol al ser única en su tipo cuenta con grandes fortalezas comerciales debido a su singularidad y originalidad. Pero a la vez tiene grandes debilidades en el sentido de que no se le da la promoción necesaria para que la población pueda conocerla en su totalidad, no solo como una mercancía si no también como parte de una cultura milenaria que ha conservado su identidad a pesar de los grandes y contantes cambios que se presentan a su alrededor.

Por otra parte la combinación de factores climáticos y geográficos poco favorables en la región son los que la limitan para desarrollar un verdadero potencial productivo, tanto en el sector agrícola, como en el ganadero, etc. Es por estas razones, que surge la necesidad de buscar estrategias alternativas para el desarrollo regional. Dentro de estas estrategias es claro que se puede promover la comercialización de la artesanía huichol, además de que se puede utilizar con fines de atractivo turístico; para de esta forma fomentar el turismo cultural en la zona norte del estado, impulsar el empleo y mejorar los niveles de vida del pueblo. Una de las grandes oportunidades que presenta la región norte del estado de Jalisco es que forman parte de la ruta wixárika o ruta de la Zona Norte, proyecto impulsado por la Secretaría de Turismo de Jalisco (Setujal) desde el año 2008, con el fin de promover el turismo regional y potencializar el comercio. La ruta dura varios días en ser recorrida, pero vale la pena hacerlo y sumergirse en su cultura y en el atractivo del entorno.

Este análisis estratégico referente a la artesanía huichol y a la región norte de Jalisco da cuenta del gran potencial de la zona en cuanto a atractivo turístico cultural se refiere y de la riqueza cultural que brinda el pueblo indígena huichol con la creación de artesanías.

Las empresas al entrar en el mercado comienzan a desenvolverse en un ambiente de competencia y rivalidad, por lo que se debe considerar a los competidores como una amenaza, se considera el poder de negociación tanto de proveedores como de compradores y las amenazas de posibles sustitutos del producto en el mercado (Porter, 1985). Por ejemplo, la intensidad de la 
rivalidad entre los competidores en el ámbito de comercialización artesanal, se presenta al momento de poner a competir la artesanía huichol con otro tipo de artesanía de la región y al ponerla a competir con sigo misma, debido a que, al ser competidores de igual tamaño, los artesanos luchan de manera vigorosa entre ellos mismos con el fin de colocar sus productos en el mercado. Por otro lado, las amenazas de entradas potenciales se presentarán sólo entre los mismos artesanos y entre las personas de la comunidad huichol que sepan hacer este tipo artesanías y que decidan entrar a este mercado de comercialización. De igual manera es necesario contemplar la amenaza de sustitutos de la artesanía huichol, pero al momento de ver la diversidad artesanal de la región se puede observar que la artesanía huichol es única en su género, por lo que no se puede remplazar una pieza hecha por los huicholes por otra que no contenga las mismas características estéticas, calidad y significado simbólico que este tipo de artesanías poseen. Referente al poder de negociación de proveedores se tomaría en cuenta en un momento dado en que la artesanía huichol se potencializara en su comercialización, y estos decidieran subir los precios de los materiales con los que se hacen, tal como la chaquira, el estambre, la cera, etc. Por último se considera el poder de negociación de los compradores debido a que son un factor fundamental al momento de fijar los precios de mercado de las artesanías, ya que muchas veces el artesano con la finalidad de vender y el cliente de comprar, comienzan un proceso de "regateo", hasta llegar a un acuerdo monetario por la artesanía, adquiriéndola muchas veces por debajo de su precio original.

En otro orden de ideas, una perspectiva líder sobre estrategia sugiere que las diferencias en el desempeño de una firma son causadas fundamentalmente por la diferencia en los recursos y las capacidades de la empresa, esta es una estrategia basada en los recursos y capacidades (Penrose, 1959). Con base en esta teoría, la producción artesanal huichol posee un sinfín de recursos y capacidades intangibles; en primer lugar porque posee significado simbólico además de que refleja sentimientos religiosos del pueblo huichol, lo cual las hace de difícil valoración económica; y en segundo lugar el conocimiento tradicional de los artesanos huicholes, que es un recurso y una capacidad incuantificable, ya que dado a este conocimiento que se es trasmitido generacionalmente es como se da lugar la creación de las artesanías.

Sin duda alguna este conocimiento tradicional añade valor agregado a la artesanía huichol, guiándola hacia una ventaja competitiva en su comercialización. Además si a este valor le agregamos la peculiaridad, la rareza y la difícil imitación de esta artesanía, dicha ventaja competitiva se potencializa en una forma considerable.

\section{Propuestas de estrategias a implementar}

Se puede ejercer un liderazgo en la comercialización de la artesanía huichol impulsando planes de desarrollo que amplíen oportunidades enfocadas especialmente al crecimiento de la 
actividad turística de la región. Es indudable que es aquí donde la participación de los gobiernos Estatales y Municipales cobrará mayor importancia, además de las instituciones como el FONART, el CDI, CONACULTA, la Dirección General de Culturas Populares, etc. contribuyendo a que los artesanos de la región consigan ese impulso que se necesita.

Según Vargas (2012) el diseño e implementación de programas de patrimonio cultural y turismo deben promover, preservar y mejorar las culturas de las comunidades, el folclore, el arte, artefactos, etc. Por lo que para la distribución y comercialización de la artesanía en la región es importante rescatar la potencialidad de la ruta wixárika para colocar y distribuir la artesanía en el mercado, esto se puede alcanzar mediante:

- $\quad$ El impulso de programas de turismo cultural en la región, donde se haga notar la riqueza en cuanto a patrimonio cultural y artístico se refiere.

- $\quad$ Ubicar puntos de venta estratégicos a lo largo de la ruta wixárika.

- Analizar la factibilidad de puntos de venta que sean taller y tienda a la vez, para que de esta manera el cliente pueda darse cuenta del proceso que se lleva a cabo en la realización de las artesanías y por otro lado pueda consumirlas.

- Ubicar hoteles y restaurantes a lo largo de la ruta wixárika y reservar un espacio para exponer las artesanías, de tal manera que estén a la vista de los visitantes de la región e incentivar su compra.

- $\quad$ Establecer tiendas dedicadas exclusivamente a las artesanías.

- Aprovechar periodos vacacionales, que es cuando la población de las zonas urbanas tienen la oportunidad de explorar la región, y llevar a cabo festivales culturales.

- Organizar ferias artesanales de fin de semana, para exponer las artesanías a la población y motivar su consumo.

Por otra parte las estrategias que se pueden seguir a nivel estatal y nacional con apoyo de las instituciones ya mencionadas y el Gobierno del Estado pueden ser:

- Impulsar acciones de publicidad en relación con los medios de comunicación, con reportajes y/o publicaciones acerca de la producción y el comercio de la artesanía.

- Invitar a los artesanos huicholes y a los de la región a ferias nacionales de productos artesanales.

- Invitar a los artesanos a eventos de promoción cultural.

- Reservar espacios, una vez al mes por ejemplo, en destinos turísticos como Puerto Vallarta Jalisco o en la ciudad de Guadalajara, para que los artesanos huicholes puedan exponer sus artesanías a los turistas y al pueblo en general, con el fin de dar a conocer su cultura y colocar sus 
productos en el mercado de una manera directa, sin la intervención de intermediarios, para que las ganancias se queden realmente con ellos.

- Organizar expo-artesanías e invitar a los artesanos huicholes a presentar sus productos.

- Buscar alianzas con cadenas comerciales para exhibir y vender artesanía huichol.

- Reservar un espacio en el aeropuerto de Guadalajara, para exponer la artesanía huichol tanto al turismo nacional como al extranjero.

Es importante resaltar que las estrategias propuestas van enfocadas básicamente a la asignación de espacios en distintos puntos estratégicos, tanto regionales como en el estado, para la exposición de la artesanía huichol y de este modo incentivar su comercialización. Lo que se pretende es presentar opciones que den oportunidad a los artesanos huicholes salir a dar a conocer parte de su cultura y de sus raíces expresadas en sus artesanías. Cabe mencionar que no se propone una industrialización de la artesanía huichol, ya que perdería valor cultural haciéndolo.

\section{Conclusiones y comentarios}

En el análisis hecho a lo largo del artículo se ha visto como las industrias culturales han tomado un papel muy importante en la preservación de la cultura e identidad nacional, además de que el impulso de las mismas es un factor a considerar como parte del crecimiento económico de los países. Retomando la interrogante planteada al principio del artículo ¿el impulso y comercialización de la artesanía huichol en la zona norte del Estado de Jalisco puede contribuir al desarrollo económico regional? La respuesta a esta pregunta de acuerdo a la perspectiva que nos dio el análisis de la producción artesanal como factor económico regional, es que si es una alternativa, dado que la actividad artesanal es una actividad que da empleo a muchos grupos domésticos dentro de la comunidad, además de que puede verse como una ruta alterna para el desarrollo comunitario.

Además, los municipios rurales de la región norte del estado de Jalisco al formar parte de la ruta wixárika presentan características idóneas para generar actividades relacionadas al turismo cultural, que de igual forma, este turismo puede contribuir a la generación de nuevos empleos, y sin duda alguna a la mejora económica de la población. Y les daría a los artesanos de la región, beneficios positivos ya que es un medio por el cual se pueden a dar a conocer sus artesanías y su cultura.

Es indudable que para alcanzar toda esta promoción y desarrollo se necesita la participación de los gobiernos municipales y estatales, además de las instituciones dedicadas al apoyo de los pueblos indígenas, contribuyendo con el impulso de programas de desarrollo regional que contemplen la producción artesanal y la cultura de la región como uno de los principales atractivos. 
Y ya que debido a que son estas instituciones los medios en los cuales los artesanos huicholes pueden apoyarse para comercializar sus artesanías, evitando la participación de intermediarios, es sumamente importante su participación activa.

Las estrategias propuestas en el artículo están pensadas con fines de promoción turística regional, donde la exposición artesanal juega un papel importante, ya que dado a esta promoción, es como se puede dar a conocer la riqueza cultural con la que cuenta la zona norte del estado. Por otra parte no se pretende imponer estas estrategias a la comunidad huichol de Jalisco, si no que son alternativas de canales de distribución que los artesanos huicholes pueden utilizar para promover su cultura, sus tradiciones, sus ideas, su cosmovisión y sus artesanías que tanto los caracterizan.

\section{Referencias}

ALBA, E. (Junio, 2008). En auge, la industria cultural en México, pese al desinterés del gobierno en su reforzamiento. La jornada Michoacán. Recuperado de www.lajornadamichoacan.com.mx

HORKHEIMER, M. Y ADORNO, TH. (1949). Dialéctica de la razón. La Industria Cultural. Iluminismo como mistificación de las masas. Buenos Aires: Sudamericana.

MÉXICO. GOBIERNO DEL ESTADO DE JALISCO (2008). Plan regional de desarrollo 2030: Región 01 Norte.

MÉXICO DESCONOCIDO. Las 10 artesanías más representativas de México. Recuperado de www.mexicodesconocido.com.mx

NOVELO, V. (1976). Artesanías y capitalismo en México. México: Instituto Nacional de Antropología e Historia.

NOVELO, V. (1993). Las artesanías en México. Tuxtla Gutiérrez, Chiapas. México: Gobierno del estado de Chiapas, Instituto Chiapaneco de Cultura.

OBSERVATORIO DE CULTURA Y ECONOMÍA (2011). Recuperado de www.culturayeconomia.org

PASTEUR, G. (2004). Artesanía prohibida. Primera edición. México: Instituto Nacional de Antropología e Historia, El Colegio de Michoacán, A.C., Universidad Autónoma de Yucatán

PENG, M. (2006). Estrategia global. Segunda edición. México: Cengage Learning Editores, S.A. de C.V.

PENROSE, E. T. (1959). The Theory of the Growth of the Firm. New York: John Wiley \& Sons.

PESCADOR, A. (2008). Las industrias culturales en un mundo globalizado. La jornada semanal. 17 de febrero de 2008. No. 676

PIEDRAS, E. (2004). ¿Cuánto vale la cultura? Contribución económica de las industrias protegidas por el derecho de autor en México. México: CONACULTA, CANIEN, SOGEN, SACM. México

PORTER, M. (1985). Competitive Advantage: Creating and sustaining superior performance. New 
York: A. Division of Simon and Shuster Inc.

SANATA, V. (2008). Industrias culturales. La jornada semanal. México en el contexto latinoamericano. 6 de enero de 2008. No. 670

RIVERA CRUZ, M., ALBERTI MANZANARES, P., VÁZQUEZ GARCÍA, V. Y MENDOZA ONTIVEROS, M. (2008). La artesanía como producción cultural susceptible de ser atractivo turístico en Santa Catarina del Monte, Texcoco. Convergencia, Vol. 15, No. 046, pp. 225-247.

SANTANDER. (Mayo de 2012). El gobierno expone a los agentes culturales las nuevas ayudas europeas. Europa press. Recuperado de www.europapress.es

UNESCO. (1997). La artesanía y el mercado internacional: comercio y codificación aduanera. Simposio UNESCO/CCI. Manila

UNESCO. (2004). Índice UNESCO: Artesanía-Turismo

VARGAS, J. (2012). Sustainable cultural and heritage tourism in regional development.

ZALLO, R. (1988). Economía de la comunicación y la cultura. Madrid: Akal

Recebido em 15.08.13

Aprovado em 10.06.14 